Proc. Indian Acad. Sci. (Math. Sci.) Vol. 114, No. 3, August 2003, pp. 225-233.

Printed in India

\title{
Characteristic properties of large subgroups in primary abelian groups
}

\author{
PETER V DANCHEV \\ Department of Mathematics, Plovdiv University, Paissii Hilendarski, 4000 Plovdiv, \\ Bulgaria \\ Current address: 13, General Kutuzov Street, Block 7, Floor 2, Flat 4, 4003 Plovdiv, \\ Bulgaria \\ E-mail: pvdanchev@yahoo.com
}

MS received 27 May 2002; revised 19 May 2004

\begin{abstract}
Suppose $G$ is an arbitrary additively written primary abelian group with a fixed large subgroup $L$. It is shown that $G$ is (a) summable; (b) $\sigma$-summable; (c) a $\Sigma$-group; (d) $p^{\omega+1}$-projective only when so is $L$. These claims extend results of such a kind obtained by Benabdallah, Eisenstadt, Irwin and Poluianov, Acta Math. Acad. Sci. Hungaricae (1970) and Khan, Proc. Indian Acad. Sci. Sect. A (1978).
\end{abstract}

Keywords. Large subgroups; summable $p$-groups; $\Sigma$ - $p$-groups; $p^{\omega+1}$-projective $p$-groups; pure-complete $p$-groups.

\section{Introduction}

The main purpose of this article is to study the relations between the structures of primary abelian groups and their large subgroups. This is a semi-expository paper, although some new results have also been proved. Thus our sections are devoted to producing a number of valuable examples of sorts of abelian groups where the structure of a $p$-group $G$ is preserved by its large subgroup $L$ and conversely. The central theorems here take the form: ' $G$ belongs to the class of abelian $p$-groups $\mathscr{K}$ if and only if $L$ belongs to $\mathscr{K}$ '. Evidently this is reminiscent of a number of well-known and documented statements of the form: ' $G$ belongs to $\mathscr{K}$ if and only if $p^{n} G$ belongs to $\mathscr{K}$ for some $n \in \mathbf{N}$ '. Our results, stated in the present paper, are clearly generalizations of such assertions for $L$ and in particular for $p^{n} G$ (see $\left.[2,4]\right)$.

In [1], Benabdallah et al proved results of the above type for the classes of:

- direct sums of cyclic $p$-groups;

- direct sums of countable $p$-groups;

- totally projective $p$-groups;

- torsion-complete $p$-groups;

- quasi-closed $p$-groups.

Later on, Khan [8] has established such an attainment for $p$-torsion abelian $\Sigma$-groups but under the additional condition that they are not separable. We shall supersede here this claim removing the condition on inseparability, i.e. by proving the attainment for arbitrary $p$-primary $\Sigma$-groups. 
We begin now with an assortment of facts (some of their proofs are quite elementary, but we include them for the sake of completeness of the exposition) that motivate this work and that are selected in the next paragraph; all notations and the terminology used are standard and will follow essentially the books of Fuchs [6] or those cited in the bibliography. Nevertheless, for the convenience of readers, we include certain crucial technical terms and notions. For instance, $p^{\omega} G=G^{1}$ is the intersection of all subgroups $p^{n} G$ for $n<\omega$. Under a large subgroup $L$ of $G$ we mean the fully invariant subgroup $L$ of $G$ with the property $G=L+B$ for every basic subgroup $B$ of $G$. In particular, $p^{n} G$ is a large subgroup of $G$ for each natural number $n$.

We add some additional background material as well from [1] and [7], necessary for our successful presentation, because of its significance to the theory.

If $L$ is an arbitrary large subgroup of a $p$-primary group $G$, then [10] and [1] independently established the explicit form of a representation for $L$, that is, $L=\sum_{k<\omega} p^{n_{k}} G\left[p^{k}\right]$, where the sequence $n_{1}, \ldots, n_{k}, \ldots$ is non-decreasing. From this it follows by folklore technical arguments that $L^{1}=G^{1}$, that $L[p]=p^{m} G[p]$ for some $m \in Z^{+}$and that $L\left[p^{2}\right]=$ $p^{j} G[p]+p^{t} G\left[p^{2}\right]$ for some $j \leq t<\omega$. However, the given representation of $L$ is not enough to determine whether $L$ inherits the structural characteristics for $G$ and conversely. That is why another approach must be demonstrated.

Next, we recall the definition and structural properties (the proofs are omitted entirely and can be found in [7]) of the so-called $A$-groups.

If $\mu$ is a limit ordinal, the class $A_{\mu}$ consists of those $p$-groups $H$ for which there is a containing totally projective $p$-group $G$ of length not exceeding $\mu$ that satisfies the following conditions: $H$ is isotype in $G, p^{\lambda}(G / H)=\left(p^{\lambda} G+H\right) / H$ whenever $\lambda<\mu$ and $G / H$ is the direct sum of a totally projective group and a divisible group. The members of the class $A_{\mu}$ are named $\mu$-elementary $A$-groups. An $A$-group is a direct sum of $\mu$ elementary $A$-groups for various limit ordinals $\mu$.

Letting $H$ denote an arbitrary reduced abelian $p$-group and $\alpha$ an arbitrary ordinal, $H$ is an $A$-group if and only if both $p^{\alpha} H$ and $H / p^{\alpha} H$ are $A$-groups.

All of these listed statements will be used extensively with the corresponding reference.

\section{Invariant properties of large subgroups}

We start with some extensions of totally projective $p$-groups, namely:

1. p-torsion A-groups. Several details on A-groups appear in [7]. For example, any $p$ torsion abelian $A$-group is an isotype subgroup of a totally projective $p$-group with special properties described in [7].

Theorem 1. Let $G$ be a reduced abelian p-group. Then $G$ is an A-group if and only if $L$ is an A-group.

Proof. First we assume that $G$ is an $A$-group. Therefore $G / G^{1}$ is a direct sum of cyclics and $G^{1}$ is an $A$-group by [7]. But from [1], $L^{1}=G^{1}$ and hence $L / L^{1} \leq G / G^{1}$. Thus $L^{1}$ is an $A$-group and $L / L^{1}$ is a direct sum of cyclics ([6], p. 110, Theorem 18.1 of $L$ Kulikov). Finally, again using [7], $L$ must be an $A$-group.

Conversely, if $L$ is an $A$-group, then so is $L^{1}=G^{1}$ by making use of [1] and [7]. On the other hand, $L / L^{1}$ is a direct sum of cyclics (see [7]) and also it is a large subgroup of $G / G^{1}$ owing to [1]. Thus we have in virtue of [1] that $G / G^{1}$ is a direct sum of cyclics, and finally [7] is applicable to get that $G$ is an $A$-group. The proof is finished. 
2. $p$-torsion $C_{\lambda}$-groups. Following Megibben, an abelian $p$-group $C$ is said to be a $C_{\lambda}$ group if $C / p^{\alpha} C$ is totally projective for all ordinals $\alpha<\lambda$.

Now, we are ready to formulate the following:

Theorem 2. Suppose $G$ is an abelian p-group and $\lambda$ is an ordinal such that $\lambda \leq$ length $(G)$. Then $G$ is a $C_{\lambda}$-group if and only if $L$ is a $C_{\lambda}$-group.

Proof. Referring to [1], we deduce that $p^{\alpha} G=p^{\alpha} L$ and $L / p^{\alpha} G$ is large in $G / p^{\alpha} G$ for each $\alpha \geq \omega$. Consequently, we can apply Theorem 4.5 of [1] to end the proof.

3. Thin p-groups. The following definition of a thin $p$-group can be referred to in [10]. The abelian $p$-group $G$ is thin if every map from a torsion-complete $p$-group to $G$ is small, that is, the kernel of this map contains a large subgroup.

We are now in a position to state the following.

Theorem 3. Let $G$ be an abelian p-group. Then $G$ is thin if and only if $L$ is thin.

Proof. If $G$ is thin, then the same property holds for $L$ as a subgroup (see [10]).

Assume now that $L$ is thin. Employing [1610], $G / L$ is a direct sum of cyclics whence thin. Consequently, taking into account [10], $G$ would be thin, as desired. The proof is finished.

4. p-torsion $\Sigma$-groups. We say that a subgroup of a $p$-torsion abelian group $G$ is high, if it is maximal with respect to $\cap G^{1}=0$. There are many non-isomorphic high subgroups of a given abelian group.

The following definition of a $\Sigma$-group is formulated in [2], namely, an abelian $p$-group $G$ is termed a $\Sigma$-group provided that some high subgroup is a direct sum of cyclics. We note that all high subgroups of a $\Sigma$-group are isomorphic because they are basic subgroups.

First of all, we list below one useful necessary and sufficient condition, obtained by us in [3], for an arbitrary abelian $p$-primary group to be a $\Sigma$-group.

Criterion. Suppose $G$ is an abelian $p$-group. Then $G$ is a $\Sigma$-group if and only if $G[p]=$ $\cup_{n<\omega} G_{n}, G_{n} \subseteq G_{n+1}$ and $G_{n} \cap p^{n} G=p^{\omega} G[p]$ for each natural number $n$.

We come now to a significant generalisation of [8].

Theorem 4. Suppose $G$ is an abelian p-group. Then $G$ is a $\Sigma$-group if and only if $L$ is a S-group.

Proof. Foremost, we recall that $p^{\omega} L=p^{\omega} G$, that there is a natural number $m$ with the property $L[p]=p^{m} G[p]$ and that $p^{n} L[p]=p^{r_{n}} G[p]$ for each $n<\omega$ and some $m \leq r_{n}<\omega$ (see [1]).

Assume now that $G$ is a $\Sigma$-group, i.e., by virtue of the above criterion, $G[p]=\cup_{n<\omega} G_{n}$, $G_{n} \subseteq G_{n+1}$ and $G_{n} \cap p^{n} G=p^{\omega} G[p]$ for every $n \in \mathbf{N}$. Hence $L[p]=\cup_{n<\omega}\left(G_{n} \cap L\right)$ and $G_{n} \cap$ $L \subseteq G_{n+1} \cap L$. Moreover, we calculate $G_{n} \cap L \cap p^{n} L=G_{n} \cap p^{n} L \subseteq G_{n} \cap p^{n} G=p^{\omega} G[p]=$ $p^{\omega} L[p]$. Our criterion leads us to the conclusion that $L$ is a $\Sigma$-group.

For the reverse inclusion, we assume that $L[p]=\cup_{n<\omega} L_{n}, L_{n} \subseteq L_{n+1}$ and $L_{n} \cap p^{n} L=$ $p^{\omega} L[p]$. Therefore $p^{m} G[p]=\cup_{n<\omega} L_{n}$. Besides, we compute, $L_{n} \cap p^{r_{n}} G[p]=L_{n} \cap p^{n} L[p]=$ $p^{\omega} L[p]=p^{\omega} G[p]$. Finally, exploiting our criterion, we detect that $p^{m} G$ is a $\Sigma$ group, i.e. the same is valid for $G$, as well (see [2]). The proof is complete after all. 
Example. (Megibben). The following construction is of a $\Sigma$-p-group which is not a $C_{\omega+1^{-}}$ group (= a pillared group in the terminology of Hill). Let $B=\oplus_{n<\omega}\left\langle p^{n}\right\rangle$ be the direct sum of cyclic groups of order $p^{n}$ and let $B^{-}$be its torsion completion. Then $B$ is pure in $B^{-}$and $B^{-} / B$ is isomorphic to $2^{\aleph_{0}}$ copies of the quasi-cyclic group $Z\left(p^{\infty}\right)$. Next, set $A=\oplus_{\lambda<2^{\aleph_{0}}}\left\langle a_{\lambda}\right\rangle$, where $\left\langle a_{\lambda}\right\rangle \cong\langle p\rangle$, a cyclic group of order $p$, for each ordinal $\lambda$. If $D$ is minimal divisible containing $A$, we have $D / A \cong B^{-} / B$. We construct a group $G$ as a subdirect sum of $B^{-}$and $D$ with kernels $B$ and $A$ respectively. So, with this in hand, it is not hard to see that $G$ is a $\Sigma$-group with a high subgroup $B$ such that $G / G^{1} \cong B^{-}$.

5. Summable p-groups. The following definition of summable $p$-primary groups is stated in [6]. We shall say that an abelian $p$-group $G$ of length $\lambda$ ( $\lambda$ is an ordinal) is summable if $G[p]=\oplus_{\alpha<\lambda} G_{\alpha}$ and $G_{\alpha} \backslash\{0\} \subseteq p^{\alpha} G \backslash p^{\alpha+1} G$ for all $\alpha<\lambda=$ length $(G)$.

Theorem 5. Suppose $G$ is a p-group. Then $G$ is summable if and only if $L$ is summable.

Proof. First, assume $G$ is summable. Thus $G[p]=\oplus_{\alpha<\lambda} G_{\alpha}$, where $G_{\alpha} \backslash\{0\} \subseteq p^{\alpha}$ $G \backslash p^{\alpha+1} G$ for each $\alpha<\lambda$. Furthermore, since $L$ is a fully invariant subgroup of $G$ and since $p^{\tau} G=p^{\tau} L$ for all ordinals $\tau \geq \omega$, we conclude $L[p]=\oplus_{\alpha<\lambda}\left(G_{\alpha} \cap L\right)$, where $\left(G_{\alpha} \cap L\right) \backslash\{0\} \subseteq p^{\alpha} L \backslash p^{\alpha+1} L$ for every $\omega \leq \alpha<\lambda$. Moreover, because $p^{n} L[p]=$ $p^{r_{n}} G[p]$ whenever $1 \leq n<\omega ; n \leq r_{n}<\omega$, we derive that $\left(G_{\alpha} \cap L\right) \backslash\{0\} \subseteq L \backslash p L$ for each $0 \leq \alpha<r_{1}$. Inductively, $\left(G_{\alpha} \cap L\right) \backslash\{0\} \subseteq p L \backslash p^{2} L$ for $r_{1} \leq \alpha<r_{2}, \ldots$, $\left(G_{\alpha} \cap L\right) \backslash\{0\} \subseteq p^{n} L \backslash p^{n+1} L$ for $r_{n} \leq \alpha<r_{n+1}$. So, by setting $\oplus_{0 \leq \alpha<r_{1}}\left(G_{\alpha} \cap L\right)=L_{0}, \ldots$, $\oplus_{r_{n} \leq \alpha<r_{n+1}}\left(G_{\alpha} \cap L\right)=L_{n}$ for every $1 \leq n<\omega$ and $G_{\alpha} \cap L=L_{\alpha}$ for every $\alpha \geq \omega$, we infer that $L[p]=\oplus_{\alpha<\lambda} L_{\alpha}$, where, for all ordinals $\alpha<\lambda, L_{\alpha} \backslash\{0\} \subseteq p^{\alpha} L \backslash p^{\alpha+1} L$. That is why, following the definition, $L$ is summable.

Now, we assume that $L$ is summable. So, $L^{1}=G^{1}$ is summable as its fully invariant subgroup. On the other hand, conforming with p. 125, Proposition 84.4 of [6], $L$ summable implies $L$ is a $\Sigma$-group. Thus Theorem 4 yields that $G$ is a $\Sigma$-group. For $H_{G}$ a high subgroup of $G$ we establish $H_{G}[p] \oplus G^{1}[p]=G[p]$. Since $H_{G}$ is a direct sum of cyclics, we may write $H_{G}[p]=\oplus_{n<\omega} H_{n}$, where $H_{n} \backslash\{0\} \subseteq p^{n} G \backslash p^{n+1} G$ because of the fact that $H_{G}$ is pure in $G$ (see, for instance, [6]). Moreover $G^{1}$ being summable ensures that $G^{1}[p]=\oplus_{\alpha<\lambda} C_{\alpha}$ with $C_{\alpha} \backslash\{0\} \subseteq p^{\alpha} G^{1} \backslash p^{\alpha+1} G^{1}=p^{\omega+\alpha} G \backslash p^{\omega+\alpha+1} G$. Therefore, we obviously observe that $G[p]=\oplus_{\beta<\omega} E_{\beta} \oplus \oplus_{\omega \leq \beta<\omega+\lambda} E_{\beta}=\oplus_{\beta<\omega+\lambda} E_{\beta}$ by putting $E_{n}=H_{n} \forall n<\omega$ and $E_{\omega+\alpha}=C_{\alpha} \forall \alpha<\lambda$. Besides, we calculate that $E_{\beta} \backslash\{0\} \subseteq p^{\beta} G \backslash p^{\beta+1} G \forall \beta<\omega+\lambda$. Finally, we extract that $G$ is summable, as stated. The proof is complete.

Example. (Hill [7]). The following is a construction of a summable $C_{\Omega}$-group that is not a direct sum of countables. For each countable ordinal $\alpha$ let $G_{\alpha}$ be the unique countable, $p$-primary group of length $\alpha$ having each of its non-zero Ulm-Kaplansky invariants equal to $\aleph_{0}$. Suppose that $\gamma<\Omega$ and that we have embedded $G_{\alpha}$ in $G_{\beta}$, for each $\alpha<\beta<\gamma$, in such a way that $p^{\lambda} G_{\beta} \cap G_{\alpha}=p^{\lambda} G_{\alpha}$ holds for all $\lambda$. If $\gamma$ is a limit ordinal, then $G_{\gamma} \cong$ $\cup_{\alpha<\gamma} G_{\alpha}$ and we have an embedding of $G_{\alpha}$ in $G_{\gamma}$ such that $p^{\lambda} G_{\beta} \cap G_{\alpha}=p^{\lambda} G_{\alpha}$ holds for $\alpha<\beta \leq \gamma$. If $\gamma$ is a non-limit ordinal, we distinguish two cases.

Case 1. $\gamma-1$ is a limit. Let $G_{\gamma-1}$ be a $p^{\gamma-1}$-high subgroup of $G_{\gamma}$.

Case 2. $\gamma-2$ exists. Let $G_{\gamma-1}$ be a direct summand of $G_{\gamma}$.

We remark, in connection with Case 1, that any $p^{\gamma-1}$-high subgroup of $G_{\gamma}$ is isomorphic to $G_{\gamma-1}$. As far as Case 2 is concerned, $G_{\gamma} \cong G_{\gamma-1}+G_{\gamma}$. Certainly, it is well-known that 
$p^{\lambda} G_{\gamma} \cap G_{\gamma-1}=p^{\lambda} G_{\gamma-1}$ for either type of embedding described above, so the condition $p^{\lambda} G_{\beta} \cap G_{\alpha}=p^{\lambda} G_{\alpha}$ continues to hold for $\alpha<\beta \leq \gamma$.

The group of interest is the union (= direct limit) of the groups $G_{\alpha}$, as $\alpha$ ranges over the countable ordinals, embedded in one another in the precise manner stated above. Let $G$ denote this group, namely $G=\cup_{\alpha<\Omega} G_{\alpha}$.

6. $\sigma$-summable p-groups and their direct sums. Following Linton and Megibben an abelian $p$-group $C$ is called $\sigma$-summable if $C[p]=\cup_{n<\omega} C_{n}, C_{n} \subseteq C_{n+1}$ and, for every positive integer $n$, there is an ordinal $\alpha_{n}$ with $C_{n} \cap p^{\alpha_{n}} C=0$ and $\alpha_{n}<$ length $(C)$. Certainly, the lengths of $\sigma$-summable $p$-groups are infinite ordinals cofinal with $\omega$.

Next, we concentrate on the following.

Theorem 6. Let $G$ be p-primary. Then $G$ is $\sigma$-summable if and only if $L$ is $\sigma$-summable.

Proof. First, since $G$ is unbounded, length $(G)=$ length $(L) \geq \omega$ (see [1]). Thus, assuming that $G$ is $\sigma$-summable, we derive that so is $L$ as its subgroup with equal length, which fact follows immediately from the definition. We only mention that the case of bounded $G$, hence bounded $L$, follows directly from [1].

Conversely, given that $L$ is $\sigma$-summable, hence $L[p]=\cup_{n<\omega} L_{n}, L_{n} \subseteq L_{n+1}$ and $L_{n} \cap$ $p^{\alpha_{n}} L=0$ for all $n \geq 0$ and some $\alpha_{n}<$ length $(G)$. Therefore, as above, $p^{m} G[p]=\cup_{n<\omega} L_{n}$. Since by [1] it holds that $p^{\alpha} G=p^{\alpha} L$ for each ordinal $\alpha \geq \omega$ or that $p^{\alpha_{n}} L[p]=p^{k_{n}} G[p]$ for $\alpha_{n}<\omega$ and some natural $k_{n} \geq \max \left(\alpha_{n}, m\right)$ because in this case $p^{\alpha_{n}} L$ is large both in $G$ and $p^{\alpha_{n}} G$, we obtain $L_{n} \cap p^{s_{n}} G=L_{n} \cap p^{\alpha_{n}} L=0$ whenever $s_{n}<$ length $(G)=$ length $\left(p^{m} G\right) \geq$ $\omega ; s_{n}=\alpha_{n} \geq \omega$ or $\omega>s_{n}=k_{n} \geq \max \left(\alpha_{n}, m\right)$.

We construct now subgroups $G_{n}$ of $G$ in the following manner: $G_{n}=\left\langle a_{n} \in G[p], L_{n}\right| a_{n} \notin$ $p^{m} G$ and if some finite sum of degrees of such generating elements $a_{n}$ 's lies in $p^{m} G$, then this sum lies in $\left.L_{n}\right\rangle$; for more details see [5] too. Bearing in mind this construction, it is apparent that $G[p]=\cup_{n<\omega} G_{n}, G_{n} \subseteq G_{n+1}$. Moreover, $G_{n} \cap p^{s_{n}} G=0$. Indeed, every element of $G_{n}$ is of the kind $\varepsilon_{1} a_{n 1}+\cdots+\varepsilon_{s} a_{n s}+b_{n}$, where $\varepsilon_{1}, \ldots, \varepsilon_{n} \geq 0$ are integers; $a_{n 1}, \ldots, a_{n s}$ are generators and $b_{n} \in L_{n}$. If $\varepsilon_{1} a_{n 1}+\cdots+\varepsilon_{s} a_{n s} \in p^{m} G$, then by definition it is in $L_{n}$, so by the above computations the studied intersection is precisely 0 . In the remaining case when $\varepsilon_{1} a_{n 1}+\cdots+\varepsilon_{s} a_{n s} \notin p^{m} G$, we find that $\varepsilon_{1} a_{n 1}+\cdots+\varepsilon_{s} a_{n s}+b_{n} \notin p^{m} G$ since $b_{n} \in L_{n} \subseteq p^{m} G$, i.e. it has height $<m \leq s_{n}$, hence the considered intersection is again equal to 0 .

This gives the desired claim and the proof is finished.

\section{PROPOSITION 7.}

Let $G$ be a direct sum of $\sigma$-summable abelian p-groups. Then so is $L$.

Proof. Write down $G=\oplus_{i \in I} G_{i}$, where all summands $G_{i}$ are $\sigma$-summable groups. Therefore $L=\oplus_{i \in I}\left(L \cap G_{i}\right)$, because it is fully invariant in $G$. But since all components $G_{i}$ are isotype in $G$, invoking [1], we deduce that $L \cap G_{i}$ are large in $G_{i}$. By what we have just argued in Theorem 6, $L \cap G_{i}$ are $\sigma$-summable groups, too. This verifies the statement, thus finishing the proof.

7. $p^{\omega+1}$-projective $p$-groups. First of all, we recall the criterion for $p^{\omega+n}$-projectivity obtained by Nunke [9] when $n \geq 0$ is an integer.

Criterion. [9]. The abelian $p$-group $G$ is $p^{\omega+n}$-projective if and only if there exists a subgroup $E \leq G\left[p^{n}\right]$ such that $G / E$ is a direct sum of cyclics. 
Clearly, subgroups of $p^{\omega+n}$-projective abelian $p$-groups are themselves $p^{\omega+n_{-}}$ projective.

The following slight modification of a classical result due to Dieudonné was proved by us in [5]; actually we have established a more general version for a larger class of abelian groups. Here we emphasize it, and freely use it in the sequel, without further comments.

Criterion.(Dieudonné, 1952). Suppose $G$ is an abelian $p$-group for which there exists a subgroup $A$ such that $G / A$ is a direct sum of cyclics. Then $G$ is a direct sum of cyclics if and only if $A[p]=\cup_{n<\omega} A_{n}, A_{n} \subseteq A_{n+1}$ and $A_{n} \cap p^{n} G=0$ for every non-negative integer $n$.

Major consequences that parallel the example listed below are the following:

1) If $G$ is a separable $p$-group such that there is $A \leq G$ with $|A|=\aleph_{0}$ and $G / A$ is a direct sum of cyclics, then $G$ is a direct sum of cyclics.

This holds easily by making use of the Dieudonné criterion since $A=\cup_{n<\omega} A_{n}$, $A_{n} \leq A_{n+1}$ and all $A_{n}$ are finite whence bounded in $G$.

2) (A result due to Fuchs, Mostowski-Sosiada; [6], p. 111, Proposition 18.3.) If $G$ is a $p$-torsion group so that there exists $A \leq G$ with $A$ a direct sum of cyclics and $G / A$ bounded, then $G$ is a direct sum of cyclics.

This follows by the usage of the Dieudonné criterion because of the fact that there is a natural number $t$ with the property $p^{t} G \subseteq A=\cup_{n<\omega} A_{n}, A_{n} \subseteq A_{n+1}$ and $A_{n} \cap p^{t+n} G \subseteq$ $A_{n} \cap p^{n} A=0$.

We now begin with the following:

Theorem 8. Suppose $G$ is an abelian p-group. Then $G$ is $p^{\omega+1}$-projective if and only if Lis $p^{\omega+1}$-projective.

Proof. As we have observed above, $G$ being $p^{\omega+1}$-projective yields that so does $L$.

To treat the converse, in view of Nunke's ctiterion stated above, we may write down $(L / E)[p]=\cup_{n<\omega}\left(L_{n} / E\right), L_{n} \subseteq L_{n+1} \leq L$ and, for each $n<\omega, L_{n} \cap p^{n} L \subseteq E$ for some $E \leq L[p]$. Certainly $L_{n} \subseteq L\left[p^{2}\right], \forall n<\omega$. Since $p^{n} L\left[p^{2}\right]=p^{t_{n}} G\left[p^{2}\right]+p^{j_{n}} G[p]$ for some $n \leq j_{n} \leq t_{n}<\omega$ (see [1]), hence $p^{t_{n}} G\left[p^{2}\right] \subseteq p^{n} L\left[p^{2}\right] \subseteq p^{j_{n}} G\left[p^{2}\right]$, we have $L_{n} \cap p^{t_{n}} G=$ $L_{n} \cap p^{t_{n}} G\left[p^{2}\right] \subseteq L_{n} \cap p^{n} L\left[p^{2}\right]=L_{n} \cap p^{n} L \subseteq E$. Thus the heights of elements from $L_{n} / E$ are bounded in $G / E$ for all $n<\omega$.

Moreover, according to [1], $(G / E) /(L / E) \cong G / L$ is a direct sum of cyclics. Finally, in the spirit of the modified variant of the classical Dieudonné criterion formulated above, that is an expansion of the classical Kulikov's criterion [6], we extract that $G / E$ is a direct sum of cyclics. That is why, in conjunction with the Nunke's criterion, $G$ is $p^{\omega+1}$ projective, as asserted. The proof is over.

Example.(Dieudonné). The following ingenious example, due to Dieudonné, demonstrates that there is a separable $p^{\omega+1}$-projective group that is not a direct sum of cyclics. Given that $C=\prod_{1 \leq k<\omega}\left\langle c_{k}\right\rangle$, where order $\left(c_{k}\right)=p$ and $C_{n}=\prod_{n<k<\omega}\left\langle c_{k}\right\rangle \forall n \geq 1$. Clearly $p C=0$, hence $C$ is a bounded direct sum of cyclic groups. For every $0 \neq x \in c_{n}+C_{n}$, let us define the elements $a_{n i}=a_{n i}(x)$ such that $p^{n} a_{n i}=x$ whenever $i \in I$ for an index set $I$. Thereby, we construct $K=\left\langle a_{n i}, C \mid n \geq 1, i \in I\right\rangle=\left\langle a_{n i} \mid n \geq 1, i \in I\right\rangle+C$. Evidently, $a_{n i} \notin C$ since $p a_{n i} \neq 0$, and moreover $|C|=2^{\aleph_{0}}>\aleph_{0}$ and $|I| \geq|C|$. Therefore $|K|=|I|$. 
First of all, we quote with some comments the following properties of the soconstructed group $K$ (see also [6], p. 16, Exercise 11):

(a) $\operatorname{order}\left(a_{n i}\right)=p^{n+1}$. For fixed $n$ and $x$, the equation $p^{n} y=x$ has at least $2 \aleph^{\aleph_{0}}$ solutions, so the index $i$ runs on $I$ with $|I| \geq 2^{\aleph_{0}}$. For instance, $a_{n j}=a_{n i}+a_{n-1, i}$ for $i \neq j$ is a guarantor for this, namely $p^{n} a_{n j}=p^{n} a_{n i}$ and order $\left(a_{n j}\right)=\operatorname{order}\left(a_{n i}\right)$. Henceforth, $\left\langle a_{n i}\right\rangle \cap\left\langle a_{n j}\right\rangle=\left\langle p^{n} a_{n i}\right\rangle=p^{n}\left\langle a_{n i}\right\rangle$. Moreover, it is self-evident that $\left\langle a_{n i}\right\rangle \cap C=\left\langle p^{n} a_{n i}\right\rangle$. In that aspect, $\left\langle a_{n i}\right\rangle \cap\left\langle a_{n+1, j}\right\rangle=0 \forall i \neq j$, and $\left\langle a_{n i}(x)\right\rangle \cap\left\langle a_{n i}(y)\right\rangle=0 \forall x \neq y \in c_{n}+C_{n}$ because $p^{n} a_{n i}(x) \neq p^{n} a_{n j}(y)$.

(b) $K / C$ is an unbounded and uncountable direct sum of cyclics. Really, $K / C=\left\langle a_{n i}\right| n \geq$ $1, i \in I\rangle+C / C \cong\left\langle a_{n i} \mid n \geq 1, i \in I\right\rangle /\left\langle a_{n i} \mid n \geq 1, i \in I\right\rangle \cap C=\left\langle a_{n i} \mid n \geq 1, i \in I\right\rangle / p^{n}\left\langle a_{n i}\right| n \geq$ $1, i \in I\rangle \cong \oplus_{n \geq 1} \oplus_{i \in I}\left[\left\langle a_{n i}\right\rangle / p^{n}\left\langle a_{n i}\right\rangle\right]$, since $\left[\left(\left\langle a_{n i}\right\rangle+C\right) / C\right] \cap\left[\left(\left\langle a_{n j}\right\rangle+C\right) / C\right]=$ $\left[\left(\left\langle a_{n i}\right\rangle+C\right) \cap\left(\left\langle a_{n j}\right\rangle+C\right)\right] / C=\left[\left(\left\langle a_{n i}\right\rangle \cap\left\langle a_{n j}\right\rangle\right)+C\right] / C=\left(\left\langle p^{n} a_{n i}\right\rangle+C\right) / C=0$.

(c) For each $0 \neq x \in c_{n}+C_{n}$ it follows that $x \in p^{n} K \backslash p^{n+1} K, x \in C \backslash p C$ and $p^{\omega} K=0$.

(d) For every $S \leq K$ such that $S$ is with elements of heights bounded in $K$ it holds that $|S \cap C|<\aleph_{0}$.

With the aid of the above Nunke's criterion and (d) it follows at once that $K$ is $p^{\omega+1}$ projective but not $p^{\omega}$-projective since otherwise $K=\cup_{n<\omega} K_{n}, K_{n} \subseteq K_{n+1}$ and $K_{n} \cap p^{n} K=$ 0 yield $C=\cup_{n<\omega}\left(K_{n} \cap C\right)$ is countable, which is obviously wrong. We indicate that $C \neq$ $K[p]$ and that $C \neq p K$.

Next, we shall find some additional properties of basic and large subgroups in $K$. In fact, $p K=\left\langle p a_{n i} \mid n \geq 1, i \in I\right\rangle$ is a large subgroup which is not a direct sum of cyclics. For a basic subgroup $B$ we have $B=\oplus_{n \geq 1} \oplus_{i \in I}\left\langle b_{n i}\right\rangle$, where $\operatorname{order}\left(b_{n i}\right)=p^{n+1}$ and $b_{n i}$ are generators which depend on $a_{n i}$; however we omit the technical details of their explicit representation. By definition, $B$ is pure in $K$, and $K / B$ is divisible that is $K=p K+B$. We also observe that $B$ is unbounded since $K$ is separable unbounded. Moreover, $K$ being $p^{\omega+1}$-projective implies that it is fully starred, hence starred. Thus, $|K|=|B| \geq 2 \aleph^{\aleph_{0}}$. Besides, $|B \cap C|=\aleph_{0}$. Indeed, writing $B_{n}=\oplus_{1 \leq k \leq n} \oplus_{i \in I}\left\langle b_{k i}\right\rangle$, we establish $B_{n} \subseteq B_{n+1}$, $B_{n} \cap p^{n} B=B_{n} \cap p^{n} K=0$ and $B=\cup_{n<\omega} B_{n}$. In lieu of (d), for each $n \geq 1$ we obtain $B_{n} \cap C$ is finite, so we are done.

Problem. Suppose $C \leq A$ such that both $C$ and $A / C$ are direct sums of $p$-cyclic groups. Then what is the algebraic structure of $A$ ?

We know that $A$ is fully starred (by Irwin-Richman), but however a more precise description is needed. For example, if $A$ is a $p$-group and $C$ is bounded at $p^{n}$, we have known via the Nunke's criterion that $A$ is $p^{\omega+n}$-projective.

8. Pure-complete $p$-groups. The following definition of a pure-complete $p$-group is stated in [6]. An abelian $p$-group $G$ is pure-complete if for every $S \leq G[p]$ there is a pure subgroup $T$ of $G$ so that $S=T[p]$.

\section{PROPOSITION 9.}

Suppose $G$ is an abelian p-group. If $G$ is pure-complete, then so is $L$.

Proof. Assume that $S \leq L[p]$. Hence $S \leq G[p]$ and $S=M[p]$ for some pure subgroup $M$ of $G$. An application of [1] assures that $M \cap L$ is large in $M$, whence it is only a routine matter to check that $M \cap L$ is pure in $L$. Finally, we may write $S=M[p] \cap L[p]=(M \cap L)[p]$, i.e. by definition $L$ is pure-complete, as claimed. The proof is completed. 


\section{COROLLARY 10.}

Let $G$ be an abelian p-group. Then $G$ is pure-complete if and only if $p^{n} G$ is pure-complete for some arbitrary fixed positive integer $n$.

Proof. The necessity follows automatically from the previous proposition via the substitution $p^{n} G=L$.

After this, the more difficult reverse question is the focus of our examination. In fact, since $p^{n} G=p\left(p^{n-1} G\right)$, a routine transfinite induction reveals that we may presume $n=1$. Now, given a subgroup $S \subseteq G[p]$ whence $S \cap p G \subseteq(p G)[p]$ and $S \cap p G=P[p]$ for some pure subgroup $P$ of $p G$. By referring [2], we infer that there is a pure subgroup $K$ of $G$ so that $p K=P$ and $K[p]=P[p]=(p K)[p]$. Therefore, $S \cap p G=(p K)[p]$. Our future aim, which we pursue, is to check that $S=T[p]$ for some pure subgroup $T$ of $G$. In fact, we construct the wanted group $T$ as follows: $T=K+(S \backslash p G) \cup\{0\}$. We need to check that $T$ is indeed a group. In fact, it is self-evident that $0 \in T$ and that if $u \in T$ then $-u \in T$. Next, let $u \in T$ and $v \in T$. Thus $u=a+b$ for $a \in K$ and $b \in(S \backslash p G) \cup\{0\}$ as well as $v=c+d$ for $c \in K$ and $d \in(S \backslash p G) \cup\{0\}$. Therefore $u+v=$ $(a+c)+(b+d)$. It is trivial that $a+c \in K$. Further, we distinguish two basic cases. First, if $b+d \in(S \backslash p G) \cup\{0\}$, there is nothing to prove. Otherwise, when $b+d \in p G$, we detect that $b+d \in S \cap p G=P[p]=K[p]$ whence $u+v \in K \subseteq T$. So, in both situations, $u+v \in T$, as needed. Besides, $T[p]=(K+(S \backslash p G) \cup\{0\})[p]=K[p]+(S \backslash p G) \cup\{0\}$ because $(S \backslash p G) \cup\{0\}=((S \backslash p G) \cup\{0\})[p]$. Henceforth $T[p]=P[p]+(S \backslash p G) \cup\{0\} \subseteq S$ and $S=(S \cap p G) \cup(S \backslash p G)=K[p] \cup((S \backslash p G) \cup\{0\})[p]=(K \cup(S \backslash p G) \cup\{0\})[p] \subseteq(K+(S \backslash p G) \cup\{0\})[p]=T[p]$. Thereby $S=T[p]$.

Finally, we shall verify that $T$ is pure in $G$. Indeed, motivated by [6], for each natural number $t$ we compute $T[p] \cap p^{t} G=S \cap p^{t} G=[K[p]+(S \backslash p G) \cup\{0\}] \cap p^{t} G=$ $[(p K)[p]+(S \backslash p G) \cup\{0\}] \cap p^{t} G=K[p] \cap p^{t} G=\left(p^{t} K\right)[p]=\left(p^{t} T\right)[p]$. This concludes the proof.

Problem. If $U$ is a pure subgroup of $L$, then does there exist a pure subgroup $V$ of $p^{n} G$ for some non-negative integer $n$ such that $V[p]=U[p]$.

If the answer is yes, $L$ being pure-complete does imply that the same is $p^{n} G$ for this $n$.

\section{Unanswered questions}

Here we quote some major problems that remain unsolved: Is it true that $G$ is a (1) direct sum of $\sigma$-summable $p$-groups; (2) $p^{\omega+n}$ projective $p$-group; (3) thick $p$-group; (4) essentially finitely indecomposable $p$-group; (5) pure-complete $p$-group; (6) semi-complete $p$ group; (7) direct sum of torsion-complete $p$-groups ([1], Problem 4); (8) Fuchs $5 p$-group; (9) Q- $p$-group; (10) $\aleph_{1}$-separable $p$-group; (11) weakly $\aleph_{1}$-separable $p$-group if and only if so is $L$ ?

However, not all properties of $G$ are inherited for $L$ and conversely. In fact, we give the following example that is our goal here: The abelian $p$-group $G$ is said to be starred (see, for instance, [10]) if it has the same power as its basic subgroup $B$. Set $L=p^{n} G$. Suppose now that $G$ is unbounded torsion-complete with a basic subgroup $B=\oplus_{0<k \leq n} \oplus_{\aleph_{1}} Z\left(p^{k}\right) \oplus \oplus_{n<k<\omega} Z\left(p^{k}\right)$ of cardinality $\boldsymbol{\aleph}_{1}$, and assume that the generalized continuum hypothesis holds. Then we claim that $G$ is starred, and $p^{n} G$ is unbounded torsion-complete (see, for example, [26]) but not starred. This is fulfilled because $p^{n} B$ is a basic subgroup of $p^{n} G$ (see [6]) and so complying with p. 29, Exercise 7 of [6] we 
compute $|G|=|B| \aleph_{0}=\aleph_{1}^{\aleph_{0}}=\aleph_{0}^{\aleph_{0}}=\aleph_{1}=|B|=\left|p^{n} G\right|=\left|p^{n} B\right|^{\aleph_{0}}>\aleph_{0}=\left|p^{n} B\right|$, that substantiates our claim.

A similar example can be given to show that $|G|>|L|$ in general. Thus the assumption that $L$ is countable does not imply that $G$ is countable.

\section{Acknowledgements}

The author is very grateful to the expert referee for helpful suggestions necessary for the careful preparation of the present manuscript.

\section{References}

[1] Benabdallah K M, Eisenstadt B J, Irwin J M and Poluianov E W, The structure of large subgroups of primary abelian groups, Acta Math. Acad. Sci. Hungaricae 21(3-4) (1970) 421-435

[2] Cutler D O, Quasi-isomorphism for infinite abelian p-groups, Pacific J. Math. 16(1) (1966) 25-45

[3] Danchev P V, Commutative group algebras of abelian $\Sigma$-groups, Math. J. Okayama Univ. 40(2) (1998) 77-90

[4] Danchev P V, Theorems of the type of Nunke for abelian groups, Compt. rend. Acad. bulg. Sci. 53(10) (2000) 5-8

[5] Danchev P V, Generalized Dieudonné criterion (to appear)

[6] Fuchs L, Infinite Abelian Groups, I and II (Moscow, Mir) (1974 and 1977) (in Russian)

[7] Hill P D, On the structure of abelian p-groups, Trans. Am. Math. Soc. 288 (1985) 505525

[8] Khan M Z, Large and high subgroups, Proc. Indian Acad. Sci. Sect. A 87(9) (1978) 177179

[9] Nunke R J, Purity and subfunctors of identity, Topics in abelian groups, Proc. Symposium, New Mexico State University (1962) pp. 121-171; (Chicago, Illinois: Scott Foresman and Co.) (1963)

[10] Richman F, Thin abelian p-groups, Pacific J. Math. 27(3) (1968) 599-606 